\title{
THE STRUCTURE OF $m$-ISOMETRIC WEIGHTED SHIFT OPERATORS
}

\author{
Belal Abdullah and Trieu LE
}

Abstract. We obtain simple characterizations of unilateral and bilateral weighted shift operators that are $m$-isometric. We show that any such operator is a Hadamard product of 2 -isometries and 3 -isometries. We also study weighted shift operators whose powers are $m$-isometric.

Mathematics subject classification (2010): 47B37, 47A65.

Keywords and phrases: $m$-isometry, weighted shift operators, Hadamard product.

\section{REFERENCES}

[1] Jim Agler, A disconjugacy theorem for Toeplitz operators, Amer. J. Math. 112 (1990), no. 1, 1-14.

[2] Jim AgLer And Mark Stankus, m-isometric transformations of Hilbert space, I, Integral Equations Operator Theory 21 (1995), no. 4, 383-429.

[3] Jim AgLeR And MARK Stankus, $m$-isometric transformations of Hilbert space, II, Integral Equations Operator Theory 23 (1995), no. 1, 1-48.

[4] Jim AgLeR AND MARK STANKUS, $m$-isometric transformations of Hilbert space, III, Integral Equations Operator Theory 24 (1996), no. 4, 379-421.

[5] Ameer Athavale, Some operator-theoretic calculus for positive definite kernels, Proc. Amer. Math. Soc. 112 (1991), no. 3, 701-708.

[6] Teresa Bermúdez, Carlos díaz Mendoza, and Antonio Martinón, Powers of $m$ isometries, Studia Math. 208 (2012), no. 3, 249-255.

[7] Teresa Bermúdez, Antonio Martinón, Vladimir Müller, and Juan Agustín Noda, Perturbation of $m$-isometries by nilpotent operators, Abstr. Appl. Anal., 2014, Art. ID 745479.

[8] Teres a Bermúdez, ANTONio MARTINón, AND EMilio Negrín, Weighted shift operators which are $m$-isometries, Integral Equations Operator Theory 68 (2010), no. 3, 301-312.

[9] FeRnANDA BOTELHO AND JAMES JAMISON, Isometric properties of elementary operators, Linear Algebra Appl. 432 (2010), no. 1, 357-365.

[10] Muneo CHO-, SCHÔICHI ÔTA, AND KôTARÔ TANAHASHI, Invertible weighted shift operators which are $m$-isometries, Proc. Amer. Math. Soc. 141 (2013), no. 12, 4241-4247.

[11] B. P. DUGGaL, Tensor product of n-isometries, Linear Algebra Appl. 437 (2012), no. 1, 307-318.

[12] Saber Elayd, An introduction to difference equations, third ed., Undergraduate Texts in Mathematics, Springer, New York, 2005.

[13] M. FAghiH-Ahmadi AND K. Hedayatian, $m$-isometric weighted shifts and reflexivity of some operators, Rocky Mountain J. Math. 43 (2013), no. 1, 123-133.

[14] S. M. PATEL, 2-isometric operators, Glas. Mat. Ser. III 37(57) (2002), no. 1, 141-145.

[15] Stefan Richter, A representation theorem for cyclic analytic two-isometries, Trans. Amer. Math. Soc. 328 (1991), no. 1, 325-349.

[16] Allen L. SHIElDS, Weighted shift operators and analytic function theory, Topics in operator theory, Amer. Math. Soc., Providence, R.I., 1974, pp. 49-128. Math. Surveys, no. 13. 